Provided for non-commercial research and educational use only. Not for reproduction or distribution or commercial use.

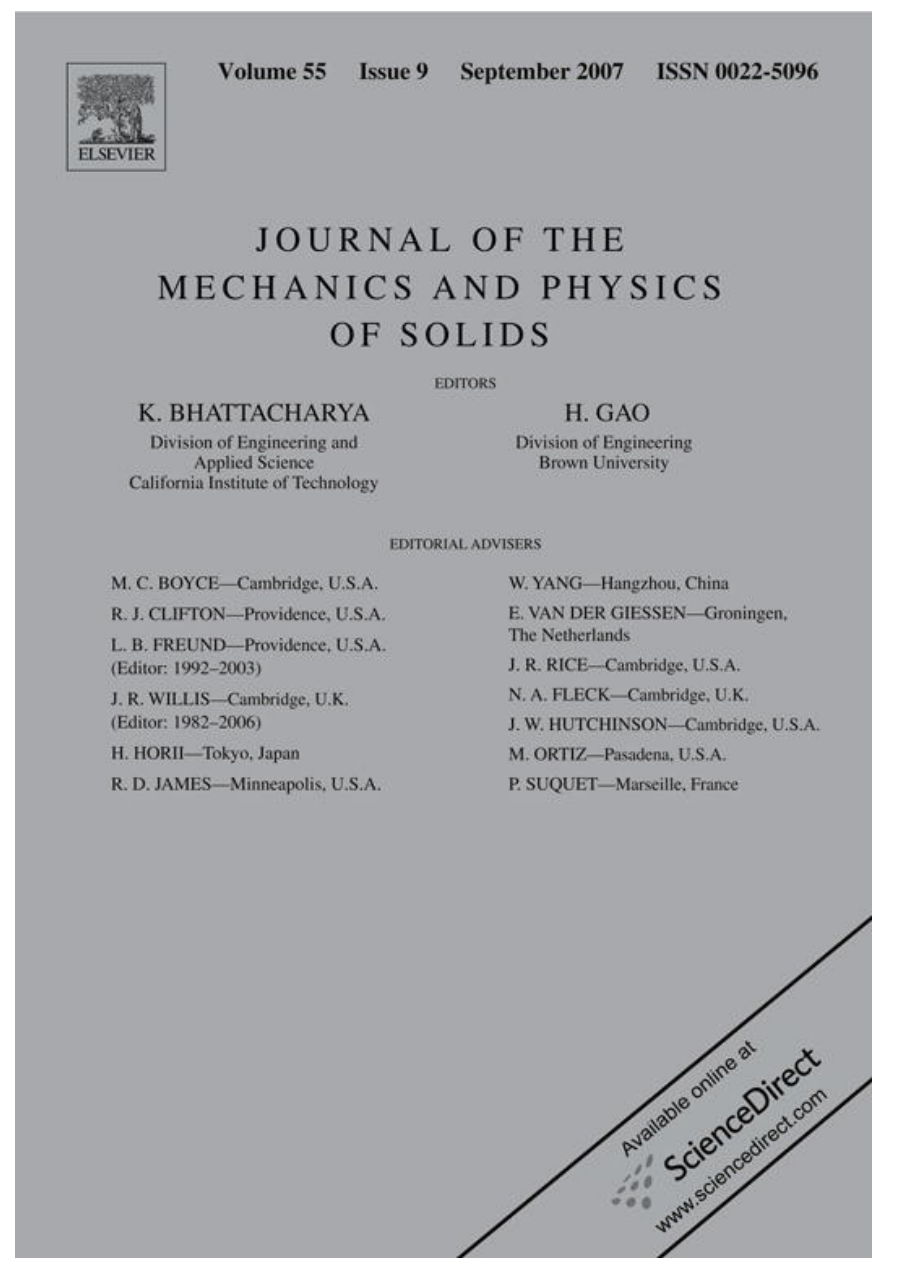

This article was originally published in a journal published by Elsevier, and the attached copy is provided by Elsevier for the author's benefit and for the benefit of the author's institution, for non-commercial research and educational use including without limitation use in instruction at your institution, sending it to specific colleagues that you know, and providing a copy to your institution's administrator.

All other uses, reproduction and distribution, including without limitation commercial reprints, selling or licensing copies or access,

or posting on open internet sites, your personal or institution's website or repository, are prohibited. For exceptions, permission may be sought for such use through Elsevier's permissions site at: 


\title{
Continuum and discrete models of dislocation pile-ups. I. Pile-up at a lock
}

\author{
R.E. Voskoboinikov*, S.J. Chapman, J.R. Ockendon, D.J. Allwright \\ OCIAM, Mathematical Institute, 24-29 St. Giles', Oxford OX1 3LB, UK
}

Received 18 October 2006; received in revised form 26 January 2007; accepted 29 January 2007

\begin{abstract}
A mathematical methodology for analysing pile-ups of large numbers of dislocations is described. As an example, the pile-up of $n$ identical screw or edge dislocations in a single slip plane under the action of an external force in the direction of a locked dislocation in that plane is considered. As $n \rightarrow \infty$ there is a well-known formula for the number density of the dislocations, but this density is singular at the lock and it cannot predict the stress field there or the force on the lock. This poses the interesting analytical and numerical problem of matching a local discrete model near the lock to the continuum model further away.
\end{abstract}

(C) 2007 Elsevier Ltd. All rights reserved.

PACS: 61.72.Lk; 61.72.Bb; 02.30.Hq

Keywords: Dislocation; Laguerre polynominals; Asymptotic expansion; Discrete; Density

\section{Introduction}

Dislocations constitute an essential part of the microstructure of crystalline solids, and their behaviour and properties directly affect the strength and toughness of structural materials (see e.g. Cahn and Haasen, 1996 and references therein). Because pure metals in most cases cannot satisfy current industrial qualifying standards, modern structural materials belong to a wide set of multi-component heterogeneous solids with developed

\footnotetext{
*Corresponding author. Tel.: + 441865270744 ; fax: + 441865270515.

E-mail address: voskoboynikov@maths.ox.ac.uk (R.E. Voskoboinikov).
} 
interfacial structure. Hence the interaction of dislocation networks with interfaces of various natures (grain boundaries, twins, stacking faults, coherent boundaries, secondary phase surfaces etc.) poses an important challenge in the context of material design and the stability of material properties under complex external influences. In particular, the presence of microstructural inhomogeneities near dislocation sources can lead to the creation of dislocation pile-ups. The stress concentration near the head of a pile-up can trigger a number of undesirable phenomena such as the initiation of Frank-Read sources, the nucleation of cracks, the delamination of a phase or stress-induced phase transformations in the adjacent piece of material. It is only through a self-consistent evaluation of the positions of dislocations in pile-ups that the stress field at the interface can be calculated and compared to the strength of the inhomogeneity, thereby allowing us to estimate the structural stability of the material.

There are two distinct analytical approaches to the description of dislocations in solids. In the discrete framework, all dislocations are treated as separate objects with definite Burgers vectors and locations. Such a discrete scenario is helpful for problems concerning a small number of dislocations interacting with each other and their surroundings but it becomes inappropriate for the treatment of a large number of closely packed dislocations for which a continuum model is more appropriate. Such a model can provide comprehensive information about the macroscopic stress but it cannot be applied at a scale at or below the separation between neighbouring dislocations in the pile-up.

This paper describes a new asymptotic methodology for analysing problems where many dislocations are present but where, nonetheless, there are regions in which the dislocations must still be represented discretely. As a prelude to a second paper which analyses the more practically important problem of pile-ups in a bimetallic solid, we will here consider the simpler case of a pile-up of screw or edge dislocations on a single slip plane against a locked dislocation, under the action of a constant applied stress. A great advantage of this configuration is that our predictions can be checked against the results of Eshelby et al. (1951), in which the problem was ingeniously reduced to finding the zeros of a Laguerre polynomial.

We will lay out our asymptotic procedure in a way that can easily be adopted in the sequel paper. In Section 2 we set up the model for an $n$-dislocation pile-up against a locked dislocation and show how it is related to the ordinary differential equation proposed in Eshelby et al. (1951). In Section 3 we take the formal limit as $n \rightarrow \infty$ to reveal the dislocation density and the approximate locations of the dislocations. However, since the density tends to infinity and zero at the head and tail of the distribution, respectively, two new asymptotic regions have to be considered separately, as shown in Sections 4 and 5. It is near the head of the distribution that the real mathematical challenge lies, as will be seen in Sections 6 and 7.

\section{Equilibrium force balance}

We consider the problem of an infinite crystalline solid with shear modulus $\mu$. We aim to find the equilibrium configuration of $n$ identical straight dislocations with Burgers vector $\mathbf{b}$ in the slip plane $y=0$ located at positions $x=x_{i}>0, i=1, \ldots, n$, under an applied stress, $\sigma$, which is independent of $z$. For the pile-up of screw dislocations $\mathbf{b}=(0,0, b)$ and the relevant component of stress is $\sigma=\sigma_{y z}$, while for edge dislocations $\mathbf{b}=(b, 0,0)$ and the relevant component of stress is $\sigma=\sigma_{x y}$. We assume that, on the $O x$ axis, $\sigma$ is given by 
$\mu \sigma_{0}(x) / 2 \pi$ if we are considering screw dislocations, and $\mu \sigma_{0}(x) / 2 \pi(1-v)$ if we are considering edge dislocations, where $v$ is the Poisson ratio. Henceforth, we will work with dimensionless variables in which distance is scaled with $b$ and stress is scaled with $\mu / 2 \pi$ for screw (respectively, $\mu / 2 \pi(1-v)$ for edge) dislocations. We will mainly be interested in dislocations under a constant external stress $\sigma_{\text {ext }}$ piling up against a locked dislocation with the same Burgers vector at the origin, for which

$$
\sigma_{0}(x)=\frac{1}{x}-\sigma_{\mathrm{ext}}
$$

but, where possible, we will simply write $\sigma_{0}(x)$. The normalised stress on $y=0$ due to such a configuration is

$$
\sigma(x)=\sum_{j=1}^{n} \frac{1}{x-x_{j}}+\sigma_{0}(x),
$$

where the first term corresponds to the stress from the dislocations themselves and the second term corresponds to the applied stress (including that from any locked dislocations).

In equilibrium the regular part of $\sigma$, obtained by subtracting the dislocation self-stress, must be zero at each $x_{i}$, giving the set of $n$ equations of equilibrium

$$
\sum_{j=1, j \neq i}^{n} \frac{1}{x_{i}-x_{j}}+\sigma_{0}\left(x_{i}\right)=0 .
$$

The goal of this paper is the asymptotic solution of (2) in the limit of large $n$, both close to and away from the lock at $x=0$. We note that the force on the lock is

$$
-\sum_{j=1}^{n} \frac{1}{x_{j}}-\sigma_{\mathrm{ext}}
$$

while the sum of (2) over $i$ gives that

$$
\sum_{j=1}^{n} \frac{1}{x_{j}}=n \sigma_{\text {ext }}
$$

Thus, the force on the lock is

$$
-(n+1) \sigma_{\mathrm{ext}}
$$

this will provide a useful check on our later calculations. This formula ${ }^{1}$ has been obtained by Eshelby et al. (1951) who proceeded by introducing the polynomial

$$
f(x)=\prod_{i=1}^{n}\left(x-x_{i}\right),
$$

\footnotetext{
${ }^{1}$ A similar formula applies to the stress on the locks in a double-ended pile-up, as can be shown using a virtual work argument, see e.g. Leibfried (1951), Hirth and Lothe (1992), Brown (2005). There the slip-line length is prescribed, whereas in our problem the slip-line length $x_{n}$ is unknown.
} 
whose zeros, $x_{i}, i=1, \ldots, n$, correspond to the dislocation positions. They observe that the logarithmic derivative of $f(x)$,

$$
\frac{f^{\prime}(x)}{f(x)}=\sum_{j=1}^{n} \frac{1}{x-x_{j}},
$$

gives the stress due to the dislocations. The force balance (2) can thus be written

$$
\lim _{x \rightarrow x_{i}}\left(\frac{f^{\prime}(x)}{f(x)}-\frac{1}{x-x_{i}}+\sigma_{0}\left(x_{i}\right)\right)=0,
$$

which reduces to

$$
\frac{f^{\prime \prime}\left(x_{i}\right)}{2 f^{\prime}\left(x_{i}\right)}+\sigma_{0}\left(x_{i}\right)=0
$$

on expanding $f(x)$ in a Taylor series near each $x_{i}$. Eshelby et al. (1951) proceed by considering the ordinary differential equation

$$
f^{\prime \prime}(x)+2 \sigma_{0}(x) f^{\prime}(x)+q(x, n) f(x)=0,
$$

where $q$ is to be determined. If $q$ can be chosen so that this equation has a polynomial solution, and $q$ is not singular at the zeros $x_{i}$ of $f(x)$, then the force balance (7) is satisfied, and the problem is solved. Finding such a $q$ for a general dislocation pile-up problem is nontrivial and is the main difficulty in applying this method. However, for the pile-up against a lock $q$ can be found, and the method of Eshelby et al. (1951) is extremely useful.

Using (1), applying the substitution $x=t /\left(2 \sigma_{\text {ext }}\right)$ and taking $q(x, n)=2 \sigma_{\text {ext }} n / x$ Eq. (8) becomes

$$
t f^{\prime \prime}(t)+(2-t) f^{\prime}(t)+n f(t)=0
$$

which is a particular case of the associated Laguerre differential equation, with the required polynomial solution

$$
f(t)=L_{n}^{1}(t)
$$

where $L_{n}^{1}(t)$ is the associated Laguerre polynomial (the other independent solution is $U(n, 2, t)$, a confluent hypergeometric function of the second kind, which is not polynomial). Thus, the positions of the dislocations in a pile-up against a lock are given by the zeros of $L_{n}^{1}(t)$.

\section{The continuum limit}

Often the only way to treat dislocation pile-ups analytically is to take a continuum limit. This can work even in the case of evolving pile-ups, see Ockendon and Ockendon (1983). In our equilibrium problem, there is more than one way to approach the continuum limit, and in this section we examine the relationship between two different approaches. We begin by using (8) to determine the dislocation density in the limit that $n \rightarrow \infty$. The substitution

$$
f(x)=v(x) \mathrm{e}^{-\int^{x} \sigma_{0}\left(x^{\prime}\right) \mathrm{d} x^{\prime}}
$$


reduces (8) to its normal form

$$
v^{\prime \prime}(x)+\kappa^{2} v(x)=0
$$

where

$$
\kappa^{2}=q(x, n)-\sigma_{0}^{\prime}(x)-\sigma_{0}^{2}(x),
$$

and the zeros of $v(x)$ coincide with those of $f(x)$, apart from perhaps additional zeros at the singularities of $\sigma_{0}$.

In the limit of large $n$ the dislocations will cover a region of $O(n)$. We therefore rescale length with $n$ by introducing the new variable $\xi=x / n$, in which (12) becomes

$$
\frac{\mathrm{d}^{2} v}{\mathrm{~d} \xi^{2}}+n^{2} \kappa^{2}(\xi, n) v=0
$$

With the assumed expansion

$$
\kappa^{2}(\xi, n) \sim \kappa_{0}^{2}(\xi)+\frac{2 \kappa_{0}(\xi) \kappa_{1}(\xi)}{n}+\cdots
$$

as $n \rightarrow \infty$, we can make the WKB expansion, see e.g. Hinch (1991),

$$
v(\xi) \sim \mathfrak{R}\left\{\mathrm{e}^{\mathrm{i} n \phi(\xi)} \sum_{k=0}^{\infty} \frac{A_{k}(\xi)}{n^{k}}\right\} .
$$

Substituting (15) in (13) and equating coefficients as $n \rightarrow \infty$ gives the eikonal equation

$$
-\left[\phi^{\prime}(\xi)\right]^{2}+\kappa_{0}^{2}(\xi)=0
$$

and leading-order amplitude equation

$$
2 A_{0}^{\prime}(\xi) \phi^{\prime}(\xi)+A_{0}(\xi) \phi^{\prime \prime}(\xi)-2 \mathrm{i} \kappa_{0}(\xi) \kappa_{1}(\xi) A_{0}(\xi)=0 .
$$

Hence

$$
v(\xi) \sim C \kappa_{0}(\xi)^{-1 / 2} \exp \left(\mathrm{in} \int_{0}^{\xi} \kappa_{0}\left(\xi^{\prime}\right) \mathrm{d} \xi^{\prime}+\mathrm{i} \int_{0}^{\xi} \kappa_{1}\left(\xi^{\prime}\right) \mathrm{d} \xi^{\prime}\right)+\text { c.c. }
$$

where c.c. denotes complex conjugate, and $C=\operatorname{Re}^{\mathrm{i} \chi}$ is a complex constant, with $R$ and $\chi$ real. The zeros of $v(\xi)$ are roots of the equation

$$
\chi+n \int_{0}^{\xi} \kappa_{0}\left(\xi^{\prime}\right) \mathrm{d} \xi^{\prime}+\int_{0}^{\xi} \kappa_{1}\left(\xi^{\prime}\right) \mathrm{d} \xi^{\prime}=\frac{\pi}{2}+k \pi, \quad k=1,2, \ldots, n .
$$

The number $N(\xi)$ of zeros in the region from the origin to $\xi$ is

$$
N(\xi)=\left\lfloor\frac{n}{\pi} \int_{0}^{\xi} \kappa_{0}\left(\xi^{\prime}\right) \mathrm{d} \xi^{\prime}+\frac{1}{\pi} \int_{0}^{\xi} \kappa_{1}\left(\xi^{\prime}\right) \mathrm{d} \xi^{\prime}+\frac{\chi}{\pi}-\frac{1}{2}\right\rfloor,
$$

where $L \cdot\rfloor$ denotes the "integer part of". Hence, to leading order, the dislocation density in the pile-up is given by

$$
\rho_{0} \sim \frac{\mathrm{d} N}{\mathrm{~d} x}=\frac{1}{n} \frac{\mathrm{d} N}{\mathrm{~d} \xi} \sim \frac{\kappa_{0}(\xi)}{\pi} .
$$


For the pile-up against a lock we have

$$
\kappa^{2}(t, n)=\frac{2 \sigma_{\mathrm{ext}}(n+1)}{x}-\sigma_{\mathrm{ext}}^{2}=\frac{2 \sigma_{\mathrm{ext}}}{\xi}-\sigma_{\mathrm{ext}}^{2}+\frac{2 \sigma_{\mathrm{ext}}}{n \xi}
$$

Hence

$$
v(\xi) \sim C\left(\frac{2 \sigma_{\text {ext }}}{\xi}-\sigma_{\text {ext }}^{2}\right)^{-1 / 4} \exp \left(2 \mathrm{i} \sin ^{-1} \sqrt{\frac{\sigma_{\text {ext }} \xi}{2}}\right) \mathrm{e}^{\mathrm{i} n \phi}+\text { c.c. }
$$

and

$$
\rho_{0}=\frac{1}{\pi}\left(\frac{2 \sigma_{\mathrm{ext}}}{\xi}-\sigma_{\mathrm{ext}}^{2}\right)^{1 / 2}
$$

where

$$
\phi=2 \sin ^{-1} \sqrt{\frac{\sigma_{\mathrm{ext}} \xi}{2}}+\sqrt{2 \sigma_{\mathrm{ext}} \xi-\sigma_{\mathrm{ext}}^{2} \xi^{2}} .
$$

A second approach to the continuum limit, which generalises easily to other pile-up problems, is to pass to a dislocation density directly in (2). This procedure is not trivial to formalise systematically in a way in which higher-order correction terms can be obtained, but it is clear that the leading-order dislocation density must satisfy (see Hirth and Lothe (1992))

$$
f_{0}^{\xi_{0}^{*}} \frac{\rho_{0}\left(\xi^{\prime}\right) \mathrm{d} \xi^{\prime}}{\xi-\xi^{\prime}}=-\hat{\sigma}_{00}(\xi)
$$

where

$$
\sigma_{0}(x)=\hat{\sigma}_{0}(\xi) \sim \hat{\sigma}_{00}(\xi)+\frac{1}{n} \hat{\sigma}_{01}(\xi)+\cdots
$$

and $\xi_{0}^{*}$ is the upper limit of the support of $\rho_{0}$, that is, $\rho_{0}(\xi)=0$ for $\xi>\xi_{0}^{*}$. Finding $\xi_{0}^{*}$ is part of the problem; it is determined from the condition that

$$
\int_{0}^{\xi_{0}^{*}} \rho_{0}\left(\xi^{\prime}\right) \mathrm{d} \xi^{\prime}=1
$$

that is, that there are exactly $n$ dislocations in the pile-up.

For the lock

$$
\hat{\sigma}_{00}(\xi)=-\sigma_{\text {ext }}, \quad \hat{\sigma}_{01}(\xi)=\frac{1}{\xi},
$$

and the appropriate solution to (24) (which is the one which tends to zero at $\xi=\xi_{0}^{*}$ and is integrable at $\xi=0)$ is of course $(23)$, with $\xi_{0}^{*}=2 / \sigma_{\text {ext }}$. Note that the stress due to the lock does not appear in the leading-order equation (24) since it is of $O(1 / n)$ in the outer region.

We note that the second approach does not require us to find the function $q$. Indeed, from (21), it allows us to find $q$ to leading order, which, in this problem, happens to be the exact result for $q$. On the other hand, since we only have the leading-order dislocation density we can only determine $\kappa$ to leading order, and therefore we know the position of the dislocations only up to $O(1 / n)$ in $\xi$ (corresponding to $O(1)$ in $x$ ), which is of the same order as their separation. Moreover, $\chi$ remains unknown. 
We now examine further the relationship between these two approaches. In particular, the following analysis will enable us to formulate an integral equation for the higher-order corrections to the dislocation density.

The key is to compare the two expressions that we have for $f$, namely the exact expression

$$
f(\xi)=n^{n} \prod_{i=1}^{n}\left(\xi-\xi_{i}\right)
$$

and the шкв approximation

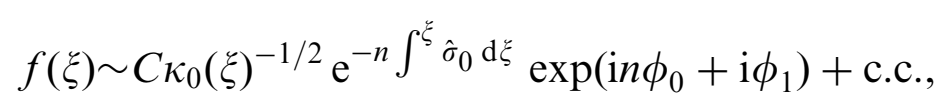

where

$$
\phi_{0}=\int_{0}^{\xi} \kappa_{0}\left(\xi^{\prime}\right) \mathrm{d} \xi^{\prime}, \quad \phi_{1}=\int_{0}^{\xi} \kappa_{1}\left(\xi^{\prime}\right) \mathrm{d} \xi^{\prime} .
$$

To approximate (27) for large $n$ we would like to take logarithms, turning the product into a sum which can then be approximated by an integral. However, the logarithm of $f$ has branch points at each zero of $f$, which are of course the positions of the dislocations, while the logarithm of the sum of two exponentials in (28) does not simplify readily.

We can get around both these problems by comparing the expressions (27) and (28) not in the region of dislocations, but in the dislocation-free zone $\xi>\xi^{*}$. In this region a similar шкв analysis holds, but the exponents of the exponentials are real rather than imaginary. We find

$$
v(\xi) \sim C_{1} \kappa_{0}(\xi)^{-1 / 2} \exp \left(\mathrm{i} n \phi_{0}+\mathrm{i} \phi_{1}\right)+C_{2} \kappa_{0}(\xi)^{-1 / 2} \exp \left(-\mathrm{i} n \phi_{0}-\mathrm{i} \phi_{1}\right),
$$

where $\phi_{0}$ and $\phi_{1}$ are still given by (29), but now $\kappa_{0}$, and $\kappa_{1}$ are positive imaginary. Since $\kappa \rightarrow \mathrm{i} \sigma_{\mathrm{ext}}$ as $\xi \rightarrow \infty$, we must have $C_{2}=0$ if we are to avoid exponential growth in the polynomial $f$. Thus,

$$
f(\xi) \sim C_{1} \kappa_{0}(\xi)^{-1 / 2} \mathrm{e}^{-n \int^{\xi} \hat{\sigma}_{0} \mathrm{~d} \xi} \exp \left(\mathrm{i} n \phi_{0}+\mathrm{i} \phi_{1}\right) .
$$

Crucially we now have a single exponential, rather than a sum of two exponentials. Now, for $\xi>\xi^{*}$, using (27) and (30) to evaluate the logarithmic derivative of $f$ we have

$$
\frac{f^{\prime}}{f}=\sum_{i=1}^{n} \frac{1}{\xi-\xi_{i}} \sim-n \hat{\sigma}_{0}+\mathrm{i} n \phi_{0}^{\prime}+\mathrm{i} \phi_{1}^{\prime}-\frac{\kappa_{0}^{\prime}}{2 \kappa_{0}}+\cdots .
$$

We want to use the Euler-Maclaurin approximation formula for the sum. Defining $g\left(\xi, \xi^{\prime}\right)=1 /\left(\xi-\xi^{\prime}\right)$, we introduce the transformation of coordinate $\zeta=\zeta\left(\xi^{\prime}\right)$ such that the dislocations are equally spaced in $\zeta$, that is $\zeta_{i}=\zeta\left(\xi_{i}\right)=i / n$. Then, setting $g\left(\xi, \xi^{\prime}\right)=G(\xi, \zeta)$ we have

$$
\sum_{i=1}^{n} G\left(\xi, \zeta_{i}\right)=n \int_{0}^{1} G(\xi, \zeta) \mathrm{d} \zeta-\frac{G(\xi, 0)}{2}+\frac{G(\xi, 1)}{2}+\cdots .
$$


Returning to the original variable, we have

$$
\sum_{i=1}^{n} \frac{1}{\xi-\xi_{i}}=n \int_{0}^{\xi^{*}} \frac{\rho\left(\xi^{\prime}\right)}{\xi-\xi^{\prime}} \mathrm{d} \xi^{\prime}-\frac{1}{2 \xi}+\frac{1}{2\left(\xi-\xi^{*}\right)}+\cdots,
$$

where $\xi^{*}=\xi_{n}$ is the position of the last dislocation, and

$$
\rho=\frac{\mathrm{d} \zeta}{\mathrm{d} \xi^{\prime}}
$$

is the dislocation density, which clearly satisfies

$$
\int_{0}^{\xi^{*}} \rho \mathrm{d} \xi^{\prime}=[\zeta]_{0}^{\xi^{*}}=\zeta\left(\xi_{n}\right)=1 .
$$

Note that there is no problem with principal value integrals, since $\xi^{\prime}=\xi$ is outside the integration region. Thus, we have

$$
n \int_{0}^{\xi^{*}} \frac{\rho\left(\xi^{\prime}\right)}{\xi-\xi^{\prime}} \mathrm{d} \xi^{\prime}-\frac{1}{2 \xi}+\frac{1}{2\left(\xi-\xi^{*}\right)}+\cdots \sim-n \hat{\sigma}_{0}+\mathrm{i} n \phi_{0}^{\prime}+\mathrm{i} \phi_{1}^{\prime}-\frac{\kappa_{0}^{\prime}}{2 \kappa_{0}}+\cdots,
$$

remembering that $\phi^{\prime}$ is positive imaginary for $\xi>\xi^{*}$, so that both sides of this equation are real-valued. So far all we have is a relationship between $\rho$ and $\phi$. However, if we now analytically continue this formula to $\xi<\xi^{*}$, we find

$$
n f_{0}^{\xi^{*}} \frac{\rho\left(\xi^{\prime}\right)}{\xi-\xi^{\prime}} \mathrm{d} \xi^{\prime}+n \pi \mathrm{i} \rho(\xi)-\frac{1}{2 \xi}+\frac{1}{2\left(\xi-\xi^{*}\right)}+\cdots \sim-n \hat{\sigma}_{0}+\mathrm{i} n \phi_{0}^{\prime}+\mathrm{i} \phi_{1}^{\prime}-\frac{\kappa_{0}^{\prime}}{2 \kappa_{0}}+\cdots
$$

Both sides of the equation are now complex, and when we equate real and imaginary parts we find two relationships between $\rho$ and $\phi$ rather than one. Expanding $\hat{\sigma}_{0}$ as in (25) and $\rho$ and $\xi^{*}$ as ${ }^{2}$

$$
\rho \sim \rho_{0}+\frac{\rho_{1}}{n}+\cdots, \quad \xi^{*} \sim \xi_{0}^{*}+\frac{\xi_{1}^{*}}{n^{2 / 3}}+\cdots,
$$

and equating real and imaginary parts of coefficients of powers of $n$ gives

$$
\begin{aligned}
& f_{0}^{\xi_{0}^{*}} \frac{\rho_{0}\left(\xi^{\prime}\right)}{\xi-\xi^{\prime}} \mathrm{d} \xi^{\prime}=-\hat{\sigma}_{00}, \\
& \pi \rho_{0}=\phi_{0}^{\prime} \\
& f_{0}^{\xi_{0}^{*}} \frac{\rho_{1}\left(\xi^{\prime}\right)}{\xi-\xi^{\prime}} \mathrm{d} \xi^{\prime}=-\hat{\sigma}_{01}+\frac{1}{2 \xi}-\frac{1}{2\left(\xi-\xi_{0}^{*}\right)}-\frac{\kappa_{0}^{\prime}}{2 \kappa_{0}}+\frac{2 a^{1 / 2}\left(-\xi_{1}^{*}\right)^{3 / 2}}{3 \pi\left(\xi-\xi_{0}^{*}\right)}, \\
& \pi \rho_{1}=\phi_{1}^{\prime},
\end{aligned}
$$

where the last term in (35) arises from the expansion

$$
\int_{\xi_{0}}^{\xi_{0}+\xi_{1} / n^{2 / 3}} \frac{\rho_{0}\left(\xi^{\prime}\right) \mathrm{d} \xi^{\prime}}{\xi-\xi^{\prime}} \sim-\frac{2 c_{0}^{1 / 2}\left(-\xi_{1}^{*}\right)^{3 / 2}}{3 n \pi\left(\xi-\xi_{0}^{*}\right)}+\cdots
$$

\footnotetext{
${ }^{2}$ The expansion for $\xi^{*}$ can be motivated either by the requirement that $\int_{\xi_{0}^{*}}^{\xi_{0}^{*}+\xi_{1}^{*} / n^{2 / 3}}\left(\rho_{0}\left(\xi^{\prime}\right) /\left(\xi-\xi^{\prime}\right)\right) \mathrm{d} \xi=O\left(n^{-1}\right)$, (using (23)) or, more readily, by the inner analysis in Section 4.
} 
where the parameter $c_{0}$ arises from the fact that, generically, as we approach the dislocation-free zone, $\kappa_{0}^{2} \rightarrow 0$ linearly as $c_{0}\left(\xi_{0}^{*}-\xi\right)$, so that $\rho_{0} \sim\left(c_{0}^{1 / 2} / \pi\right)\left(\xi_{0}^{*}-\xi\right)^{1 / 2}$. For the pile-up against a lock, $c_{0}=\sigma_{\text {ext }}^{3} / 2$.

We see that Eq. (33) coincides with (24), while Eq. (34) is just (21). Crucially though, this approach allows us to construct an equation for the first-order correction to the density, which is Eq. (35); from this equation we can determine $\kappa_{1}$ even if we do not know $q$.

For the pile-up against a lock we have

$$
\begin{aligned}
f_{0}^{\xi^{*}} \frac{\rho_{1}\left(\xi^{\prime}\right)}{\xi-\xi^{\prime}} \mathrm{d} \xi^{\prime} & =-\frac{1}{\xi}+\frac{1}{2 \xi}-\frac{1}{2\left(\xi-2 / \sigma_{\mathrm{ext}}\right)}-\frac{1}{2 \xi\left(\sigma_{\mathrm{ext}} \xi-2\right)}+\frac{2^{1 / 2} \sigma_{\mathrm{ext}}^{3 / 2}\left(-\xi_{1}^{*}\right)^{3 / 2}}{3 \pi\left(\xi-2 / \sigma_{\mathrm{ext}}\right)} \\
& =-\frac{1}{4 \xi}+\left(-\frac{3}{4}+\frac{2^{1 / 2} \sigma_{\mathrm{ext}}^{3 / 2}\left(-\xi_{1}^{*}\right)^{3 / 2}}{3 \pi}\right) \frac{1}{\left(\xi-2 / \sigma_{\mathrm{ext}}\right)} .
\end{aligned}
$$

The normalisation condition on $\rho_{1}$ is determined by the $O\left(n^{-1}\right)$ balance in Eq. (31). Expanding (31) we find

$$
\int_{0}^{\xi_{0}^{*}} \rho_{1} \mathrm{~d} \xi \sim-\int_{\xi_{0}}^{\xi_{0}+\xi_{1} / n^{2 / 3}} \rho_{0}\left(\xi^{\prime}\right) \mathrm{d} \xi^{\prime} \sim \frac{2^{1 / 2} \sigma_{\text {ext }}^{3 / 2}\left(-\xi_{1}^{*}\right)^{3 / 2}}{3 \pi} .
$$

The right-hand side of (37) generates the particular solution

$$
\rho_{1}=-\frac{1}{4} \delta(\xi)+\left(-\frac{3}{4}+\frac{2^{1 / 2} \sigma_{\mathrm{ext}}^{3 / 2}\left(-\xi_{1}^{*}\right)^{3 / 2}}{3 \pi}\right) \delta\left(\xi-2 / \sigma_{\mathrm{ext}}\right) .
$$

To this solution we must add a multiple of the eigensolution

$$
\frac{1}{\sqrt{\xi\left(2 / \sigma_{\mathrm{ext}}-\xi\right)}}
$$

in order to satisfy (38), so that the full solution for $\rho_{1}$ is given by

$$
\rho_{1}=\frac{1}{\pi \sqrt{\xi\left(2 / \sigma_{\text {ext }}-\xi\right)}}-\frac{1}{4} \delta(\xi)+\left(-\frac{3}{4}+\frac{2^{1 / 2} \sigma_{\mathrm{ext}}^{3 / 2}\left(-\xi_{1}^{*}\right)^{3 / 2}}{3 \pi}\right) \delta\left(\xi-2 / \sigma_{\text {ext }}\right) .
$$

Of course, the $\delta$-functions evaluate to zero in the outer region; they are just the way the outer region "sees" the inner regions near $\xi=0$ and $\xi=\xi^{*}$. Since $\rho_{1}=\phi_{1}^{\prime} / \pi=\kappa_{1} / \pi$ we see that (39) agrees with (22).

As we have already intimated, this outer "continuum" approximation breaks down at the head of dislocation pile-up, at which it predicts an infinite dislocation density and stress, and also at the boundary of a dislocation-free region (which corresponds to a turning point in (13)). In the vicinity of these regions inner approximations need to be developed, in which the dislocations are separated by order-one distances and the WKB approximation is not used in the equation for $v$.

\section{Inner solution near the turning point}

As mentioned above, generically as we approach the dislocation-free zone $\kappa_{0}^{2} \rightarrow 0$ linearly as $c_{0}\left(\xi_{0}^{*}-\xi\right)$ so that $\rho_{0} \sim\left(c_{0}^{1 / 2} / \pi\right)\left(\xi_{0}^{*}-\xi\right)^{1 / 2}$. We define the inner variable as $\xi-$ 
$\xi_{0}^{*}=n^{-2 / 3} \tau$ to give

$$
v^{\prime \prime}(\tau)-c_{0} \tau v(\tau)=0
$$

where ' represents $\mathrm{d} / \mathrm{d} \tau$. To match with the solution (30) in the dislocation-free region we need $v$ to decay as $\tau \rightarrow \infty$, so that

$$
v(\tau)=\text { const. } \times \operatorname{Ai}\left(c_{0}^{1 / 3} \tau\right),
$$

where Ai is the Airy function. As $\tau \rightarrow-\infty$ this is

$$
v(\tau) \sim \text { const. } \times \frac{1}{(-\tau)^{1 / 4}} \cos \left(\frac{2 c_{0}^{1 / 2}(-\tau)^{3 / 2}}{3}-\frac{\pi}{4}\right) .
$$

As $\xi \rightarrow 2 / \sigma_{\text {ext }}$ the outer solution (18) is given by

$$
\text { real const. } \times \frac{1}{(-\tau)^{1 / 4}} \cos \left(-\frac{2 c_{0}^{1 / 2}(-\tau)^{3 / 2}}{3}+\chi+n \int_{0}^{\xi_{0}^{*}} \kappa_{0}\left(\xi^{\prime}\right) \mathrm{d} \xi^{\prime}+\int_{0}^{\xi_{0}^{*}} \kappa_{1}\left(\xi^{\prime}\right) \mathrm{d} \xi^{\prime}\right) .
$$

Matching this with (41) gives the previously unknown phase

$$
\chi=m \pi+\frac{\pi}{4}-n \int_{0}^{\xi_{0}^{*}} \kappa_{0}\left(\xi^{\prime}\right) \mathrm{d} \xi^{\prime}-\int_{0}^{\xi_{0}^{*}} \kappa_{1}\left(\xi^{\prime}\right) \mathrm{d} \xi^{\prime}
$$

where $m \in \mathbb{Z}$.

For the pile-up against a lock,

$$
\int_{0}^{\xi_{0}^{*}} \kappa_{0}\left(\xi^{\prime}\right) \mathrm{d} \xi^{\prime}=\pi, \quad \int_{0}^{\xi_{0}^{*}} \kappa_{1}\left(\xi^{\prime}\right) \mathrm{d} \xi^{\prime}=\pi
$$

and

$$
\chi=\pi / 4 \text {. }
$$

We developed the inner expansion above for a generic boundary between a region of dislocations and a dislocation-free zone, using the pile-up against a lock as an example. We discuss here briefly why we believe that (40) will generically be the leading-order inner equation.

If the outer expansion of $\kappa^{2}$ is, as in (14),

$$
\kappa^{2}=k_{0}+\frac{k_{1}}{n}+\cdots
$$

and if, in the vicinity of the turning point,

$$
k_{0} \sim c_{0}\left(\xi_{0}^{*}-\xi^{*}\right), \quad k_{1} \sim c_{1},
$$

where $c_{i}$ are constants, then the inner limit of the outer expansion of $\kappa^{2}$ is

$$
\frac{1}{n^{2 / 3}} c_{0} \tau+\frac{c_{1}}{n}+\cdots
$$

The important point is that the leading-order term, of $O\left(n^{-2 / 3}\right)$, arises solely from the leading-order outer expansion. Matching with the outer limit of the inner expansion indicates that the leading-order inner expansion of $\kappa^{2}$ is simply the inner limit of the leading-order outer expansion. For this not to be the case would require either (i) that $k_{1}$ is singular with $k_{1} \sim c_{1}\left(\xi_{0}^{*}-\xi^{*}\right)^{-1 / 2}$ as $\xi \rightarrow \xi_{0}^{*}$ so that the inner limit of the outer 
expansion of $\kappa^{2}$ is

$$
\frac{1}{n^{2 / 3}} c_{0} \tau+\frac{c_{1}}{\tau^{1 / 2} n^{1 / 2}}+\cdots
$$

or (ii) that (14) is replaced by an expansion of the form

$$
\kappa^{2} \sim k_{0}+\frac{k_{1}}{n^{2 / 3}}+\cdots
$$

Both of these seem unlikely.

\section{Inner solution near the lock}

The dislocation density tends to infinity as $\xi \rightarrow 0$, and the continuum approximation predicts an infinite stress on the lock at $\xi=0$. To regularise this we need to introduce an inner region in the vicinity of $\xi=0$ in which the dislocations are not smeared into a density and their individual positions are determined. As $\xi \rightarrow 0, \rho$ tends to infinity as $1 / \xi^{1 / 2}$, so that the spacing of the dislocations is $O\left(n^{-2}\right)$ (in terms of $\xi$, or $O\left(n^{-1}\right)$ in terms of the original variable $x$ ) in the inner region (recall that the spacing in the continuum outer region is $O\left(n^{-1}\right)$ in $\xi$ or $O(1)$ in $\left.x\right)$.

This motivates the introduction of the inner variable $\eta=n^{2} \xi=n x$, in which (13) becomes

$$
v^{\prime \prime}(\eta)+\left(\frac{2 \sigma_{\mathrm{ext}}}{\eta}-\frac{\sigma_{\mathrm{ext}}^{2}}{n^{2}}+\frac{2 \sigma_{\mathrm{ext}}}{n \eta}\right) v(\eta)=0,
$$

where' represents $\mathrm{d} / \mathrm{d} \eta$. At leading order

$$
v^{\prime \prime}(\eta)+\frac{2 \sigma_{\mathrm{ext}}}{\eta} v(\eta)=0
$$

with solution

$$
v(\eta)=\sqrt{\eta}\left(A J_{1}\left(\sqrt{8 \sigma_{\mathrm{ext}} \eta}\right)+B Y_{1}\left(\sqrt{8 \sigma_{\mathrm{ext}} \eta}\right)\right),
$$

where $J_{1}$ and $Y_{1}$ are Bessel functions of order one. Now the total stress at any point (including the applied stress) is

$$
\sigma=\frac{v^{\prime}(x)}{v(x)}=n \frac{v^{\prime}(\eta)}{v(\eta)}
$$

As $\eta \rightarrow 0$ this stress must be the stress due to the locked dislocation, i.e.

$$
\frac{v^{\prime}(\eta)}{v(\eta)} \sim \frac{1}{\eta} \quad \text { as } \eta \rightarrow 0
$$

Since

$$
J_{1}(s) \sim \frac{s}{2}-\frac{s^{3}}{16}+\cdots, \quad Y_{1}(s) \sim-\frac{2}{\pi s}+\frac{s \log s}{\pi}+\cdots \quad \text { as } s \rightarrow 0,
$$


$v^{\prime} / v$ is bounded at the origin unless $B=0$, in which case (46) is satisfied. Thus, the leadingorder position of the dislocations in the inner region are given by

$$
\eta_{i}=\frac{\alpha_{i}^{2}}{8 \sigma_{\mathrm{ext}}},
$$

corresponding to

$$
x_{i}=\frac{\alpha_{i}^{2}}{8 n \sigma_{\mathrm{ext}}},
$$

where $\alpha_{i}$ are the zeros of $J_{1}$, so that $\alpha_{i} \sim \pi(i+1 / 4)$ as $i \rightarrow \infty$. As $\eta \rightarrow \infty$,

$$
v \sim \frac{A \eta^{1 / 4}}{\sqrt{\pi}\left(2 \sigma_{\mathrm{ext}}\right)^{1 / 4}} \cos \left(\sqrt{8 \sigma_{\mathrm{ext}} \eta}-\frac{3 \pi}{4}\right) .
$$

As $\xi \rightarrow 0$ in (22),

$$
v \sim \operatorname{Re}^{\mathrm{i} \chi} \frac{\xi^{1 / 4}}{\left(2 \sigma_{\text {ext }}\right)^{1 / 4}} \mathrm{e}^{\mathrm{i} n \sqrt{8 \sigma_{\text {ext }} \xi}}+\text { c.c. }
$$

We see that (48) and (49) match providing $\chi=\pi / 4$, in agreement with (42).

We observe that the position of the dislocations in the inner region in this problem is determined wholly by the local analysis near $x=0$; the behaviour at the origin is sufficient to eliminate one constant from the inner solution (45), and the phase matching for $\chi$ through the outer region to the turning point is automatic.

Using the properties of $J_{1}$ we can now calculate the force on the lock, which is

$$
n \lim _{\eta \rightarrow 0}\left(\frac{v^{\prime}}{v}-\frac{1}{\eta}\right)=-n \sigma_{\mathrm{ext}},
$$

and this agrees with our exact result (3) to lowest order. The discrepancy between (3) and (50) can be easily resolved by carrying the expansion (14) to second order, but we will not do this here.

The inner analysis presented above crucially required us to know $q$ in the inner region. In contrast to the discussion after (42), we have no reason to believe that the leading-order inner expansion of $q$ should be the inner limit of the leading-order outer expansion in the vicinity of the lock. It happens to be true in this example, but when we consider other configurations (such as the pile-up of dislocations at a bimetallic interface to be considered in II), we will not know what the inner expression for $q$ is (and indeed in II the singular behaviour of the outer expansion for $q$ means that the inner expansion cannot be simply the inner limit of the outer expansion), and we will need a different method of solution in the inner region.

\section{Formulation of an inner discrete problem near the lock}

Let us go back to our exact expression for the stress,

$$
\sigma=\sum_{j=1}^{n} \frac{1}{x-x_{j}}+\frac{1}{x}-\sigma_{\mathrm{ext}} .
$$

We first need to split the sum into those dislocations which are "in the inner region", and those which are "in the outer region". Of course, as in any problem in matched 
asymptotics, there is not a sharp jump from inner to outer but a smooth transition. We split the sum at dislocation $k$, where $x_{k}$ lies in the overlap region between the inner and outer regions, that is, $1 / n \ll x_{k} \ll 1$.

For the dislocations with $x_{i} \leqslant x_{k}$ we use the inner scaling $x_{i}=n^{-1} \eta_{i}$, while for those with $x_{i}>x_{k}$ we use the outer scaling $x_{i}=n \xi_{i}$. Then, in the inner region with $x=n^{-1} \eta$ we have

$$
\begin{aligned}
\sigma & =\sum_{j=1}^{k} \frac{1}{x-x_{j}}+\sum_{j=k+1}^{n} \frac{1}{x-x_{j}}+\frac{1}{x}-\sigma_{\mathrm{ext}} \\
& =n \sum_{j=1}^{k} \frac{1}{\eta-\eta_{j}}+\frac{1}{n} \sum_{j=k+1}^{n} \frac{1}{\eta / n^{2}-\xi_{j}}+\frac{n}{\eta}-\sigma_{\mathrm{ext}} .
\end{aligned}
$$

The second sum can be approximated as an integral using Euler-Maclaurin summation as in Section 3. We find

$$
\frac{1}{n} \sum_{j=k+1}^{n} \frac{1}{\eta / n^{2}-\xi_{j}} \sim \int_{\xi_{k+1}}^{\xi^{*}} \frac{\rho\left(\xi^{\prime}\right) \mathrm{d} \xi^{\prime}}{\eta / n^{2}-\xi^{\prime}}+\frac{1}{2 n\left(\eta / n^{2}-\xi^{*}\right)}+\frac{1}{2 n\left(\eta / n^{2}-\xi_{k+1}\right)}+\cdots .
$$

Note again that there is no problem with principal value integrals, since $\xi^{\prime}=\eta / n^{2}$ is outside the integration region. Now from (24) we know that the outer density satisfies

$$
f_{0}^{\xi^{*}} \frac{\rho_{0}\left(\xi^{\prime}\right)}{\xi-\xi^{\prime}} \mathrm{d} \xi^{\prime}=\sigma_{\text {ext }} .
$$

Combining this with (51) we find that, to lowest order,

$$
\frac{1}{n} \sum_{j=k+1}^{n} \frac{1}{\eta / n^{2}-\xi_{j}} \sim \sigma_{\mathrm{ext}}-f_{0}^{\xi_{k+1}} \frac{\rho_{0}\left(\xi^{\prime}\right) \mathrm{d} \xi^{\prime}}{\eta / n^{2}-\xi^{\prime}} .
$$

Now as $\xi \rightarrow 0$,

$$
\rho_{0} \sim \frac{\sigma_{\mathrm{ext}}}{\pi} \frac{\sqrt{\xi_{0}^{*}}}{\sqrt{\xi}}-\frac{\sigma_{\mathrm{ext}}}{2 \pi} \frac{\sqrt{\xi}}{\sqrt{\xi_{0}^{*}}}+\cdots
$$

Hence, since $\xi_{k+1} \ll 1$,

$$
\begin{aligned}
f_{0}^{\xi_{k+1}^{k}} \frac{\rho_{0}\left(\xi^{\prime}\right) \mathrm{d} \xi^{\prime}}{\eta / n^{2}-\xi^{\prime}} & \sim \frac{\sigma_{\mathrm{ext}} \sqrt{\xi_{0}^{*}}}{\pi} f_{0}^{\xi_{k+1}} \frac{\left(\xi^{\prime}\right)^{-1 / 2} \mathrm{~d} \xi^{\prime}}{\eta / n^{2}-\xi^{\prime}} \\
& \sim \frac{n \sigma_{\mathrm{ext}} \sqrt{\xi_{0}^{*}}}{\pi} f_{0}^{\eta_{k+1}} \frac{\left(\eta^{\prime}\right)^{-1 / 2} \mathrm{~d} \eta^{\prime}}{\eta-\eta^{\prime}} \\
= & \frac{n \sigma_{\mathrm{ext}} \sqrt{\xi_{0}^{*}}}{\pi} \frac{2}{\sqrt{\eta}} \tanh ^{-1}\left(\sqrt{\frac{\eta}{\eta_{k+1}}}\right) \\
& \sim \frac{n \sigma_{\mathrm{ext}} \sqrt{\xi_{0}^{*}}}{\pi} \frac{2}{\sqrt{\eta_{k+1}}} .
\end{aligned}
$$

Thus, in the inner region,

$$
\sigma \sim n \sum_{j=1}^{k} \frac{1}{\eta-\eta_{j}}+\frac{n}{\eta}-\frac{n \sigma_{\mathrm{ext}} \sqrt{\xi_{0}^{*}}}{\pi} \frac{2}{\sqrt{\eta_{k+1}}} .
$$


Evaluating this at the dislocations gives the inner force balance equation as

$$
\sum_{j=1, j \neq i}^{k} \frac{1}{\eta_{i}-\eta_{j}}+\frac{1}{\eta_{i}} \sim \frac{\sigma_{\mathrm{ext}} \sqrt{\xi_{0}^{*}}}{\pi} \frac{2}{\sqrt{\eta_{k+1}}},
$$

where $i \ll k$. This condition on $i$ means that we cannot solve (53) directly as it stands, since we have $k$ unknowns and many fewer than $k$ equations. However, remembering that $k$ is large we find that the leading-order inner equation is

$$
\sum_{j=1}^{\infty} \frac{1}{\eta_{i}-\eta_{j}}+\frac{1}{\eta_{i}}=0
$$

In the inner region the equations for the dislocation positions are independent of the external force $\sigma_{\text {ext }}$. The only information the inner dislocations have concerning the external force $\sigma_{\text {ext }}$ comes from the matching condition, which we now derive.

Subtracting (54) from (53) we find that the late terms satisfy

$$
\sum_{j=k+1}^{\infty} \frac{1}{\eta_{i}-\eta_{j}} \sim-\frac{\sigma_{\mathrm{ext}} \sqrt{\xi_{0}^{*}}}{\pi} \frac{2}{\sqrt{\eta_{k+1}}}
$$

To match with the singularity in the outer dislocation density the behaviour as $i \rightarrow \infty$ must be given by $\eta_{i} \sim B i^{2}$, for some constant $B$ (in fact the analysis of Section 5 has forewarned us of this, but, as shown in Section 7 , this result can be inferred directly from (54)). This asymptotic behaviour implies

$$
\sum_{j=k+1}^{\infty} \frac{1}{\eta_{i}-\eta_{j}} \sim-\frac{1}{B} \int_{k+1}^{\infty} i^{-2} \mathrm{~d} i=-\frac{1}{B(k+1)}=-\frac{1}{\sqrt{B \eta_{k+1}}},
$$

which is consistent with (55) if

$$
B=\frac{\pi^{2}}{4 \sigma_{\mathrm{ext}}^{2} \xi_{0}^{*}}=\frac{\pi^{2}}{8 \sigma_{\mathrm{ext}}} .
$$

Let us check that the dislocation densities in the inner and outer regions match. From (52) we deduce that

$$
\frac{2 n \sigma_{\mathrm{ext}} \sqrt{\xi_{0}^{*}}}{\pi} \xi_{i}^{1 / 2} \sim i, \quad \text { i.e. } \xi_{i} \sim \frac{i^{2} \pi^{2}}{4 n^{2} \sigma_{\mathrm{ext}}^{2} \xi_{0}^{*}},
$$

as $i \rightarrow 0$, so that in order to match we need

$$
\eta_{i} \sim \frac{i^{2} \pi^{2}}{4 \sigma_{\mathrm{ext}}^{2} \xi_{0}^{*}}
$$

as $i \rightarrow \infty$ in agreement with (57).

Now let us consider the problem of how to solve (54) numerically, since for more general problems we are unlikely to be able to find an exact analytical solution. Clearly to solve numerically we must truncate the infinite system of equations, replacing the late terms by their asymptotic behaviour. However, if we simply truncate the sum as in (53), the resulting equation is accurate only for $i \ll k$, leading to a problem with more unknowns than equations. The key is to choose a large integer $N$ and replace $\eta_{i}$ in (54) with its asymptotic 


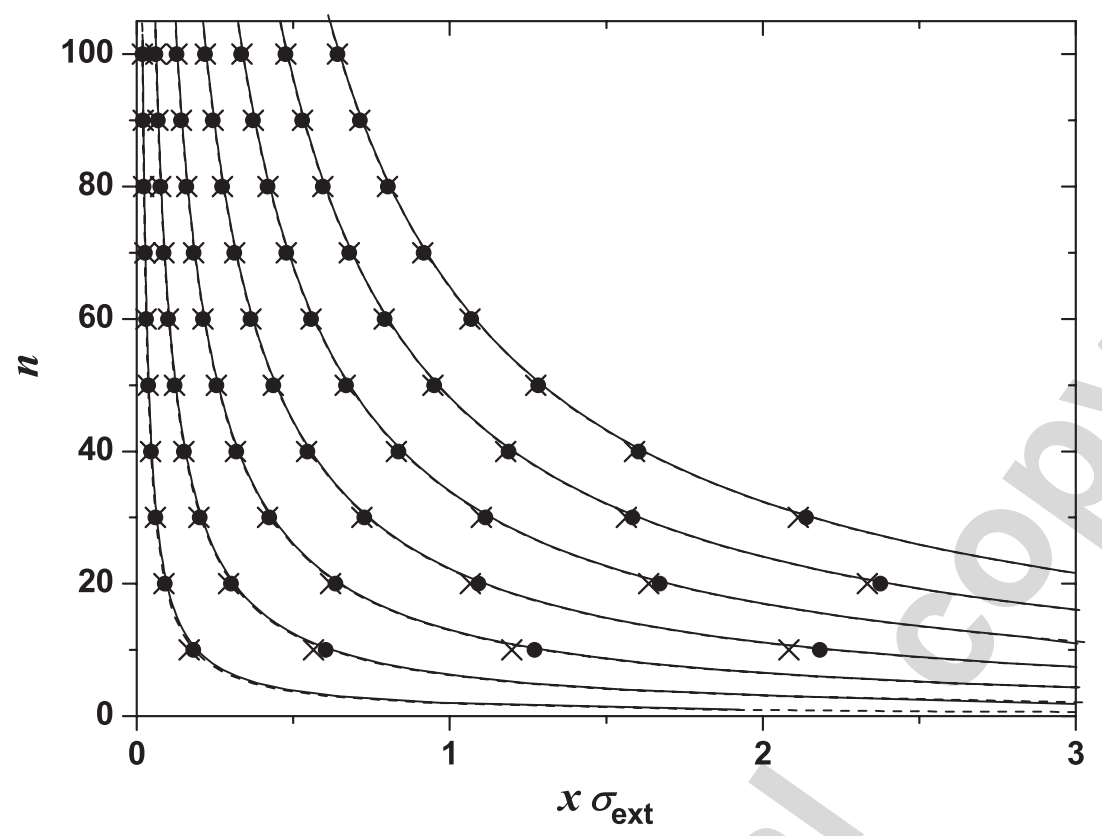

Fig. 1. The normalised dislocation positions $x_{i} \sigma_{\mathrm{ext}}, i=1 \ldots n$, as a function of the total number of dislocations $n$ subject to an applied stress $\sigma_{\text {ext }}$ and the lock at the origin. Recall that $x$ has been made dimensionless with $b$ and $\sigma_{\text {ext }}$ with $\mu / 2 \pi$ or $\mu / 2 \pi(1-v)$ for the cases of screw and edge dislocations: for $\sigma_{\text {ext }}=10^{-3}, x \sigma_{\text {ext }}=1$ corresponds to a dimensional value of $10^{3} b$. For $n=30, \ldots, 100, n=20$ and 10 positions of first seven, six and four dislocations, respectively, are displayed. Two discrete calculations are shown: the crosses are the positions calculated numerically from the full problem (2); the dots are those calculated by solving the discrete inner equation (53) as discussed above with $N=10$ and $k=20$. The solid curve shows the analytical solution to the inner equation (47), while the dashed curve (which lies almost on top of the solid curve) shows the asymptotic approximation to this for large $i$ in which $\alpha_{i} \sim \pi(i+1 / 4)$. When there are only 10 dislocations the position of the second and higher dislocations is better approximated using a uniform combination of the inner and outer expansions.

behaviour (58) for $i>N$, leading to $N$ equations in $N$ unknowns. The infinite sums can then be truncated using (55), but $k$ must be chosen to be much bigger than $N$.

In Fig. 1 we show a comparison of the predicted positions of the leading seven dislocations using our various methods of solution for different values of $n$. The crosses correspond to a numerical solution of the full problem (2). The dots correspond to solving the discrete inner equation (53) as discussed above with $N=10$ and $k=20$, that is, we solve for 10 dislocations in the inner region. The solid curve shows the analytical solution to the inner equation (47), while the dashed curve shows the asymptotic approximation to this for large $i$ in which $\alpha_{i} \sim \pi(i+1 / 4)$. When there are only 10 dislocations the position of the second and higher dislocations is not really in the inner region, and is better approximated using a uniform combination of the inner and outer expansions.

\section{Uniqueness of the discrete inner solution}

We know that the dislocation positions in the inner region should be proportional to the squares of the zeros of $J_{1}$. Let us now see how we can deduce this fact from our discrete inner equation (54). 
We first note that (54) implies that

$$
\sum_{j=1}^{\infty} \frac{1}{\eta_{j}}=c
$$

is convergent. Now, following the approach of Section 2, we define

$$
f(\eta)=\eta \prod_{j=1}^{\infty}\left(1-\frac{\eta}{\eta_{j}}\right)
$$

where the product is absolutely convergent. Then

$$
\begin{aligned}
& \frac{f^{\prime}}{f}=\frac{1}{\eta}+\sum_{j=1}^{\infty} \frac{1}{\eta-\eta_{j}}, \\
& \frac{f^{\prime \prime}}{f}-\left(\frac{f^{\prime}}{f}\right)^{2}=-\frac{1}{\eta^{2}}-\sum_{j=1}^{\infty} \frac{1}{\left(\eta-\eta_{j}\right)^{2}} .
\end{aligned}
$$

Now, as $\eta \rightarrow 0$,

$$
\frac{f^{\prime}}{f}=\frac{1}{\eta}-c+O(\eta),
$$

so that

$$
\frac{f^{\prime \prime}}{f}=-\frac{2 c}{\eta}+O(1) .
$$

Similarly, as $\eta \rightarrow \eta_{i}$

$$
\frac{f^{\prime}}{f}=\frac{1}{\eta-\eta_{i}}+O\left(\eta-\eta_{i}\right),
$$

(in which the absence of an $O(1)$ term results from $\eta_{i}$ satisfying (54)), so that

$$
\frac{f^{\prime \prime}}{f}=O(1) \text {. }
$$

Thus,

$$
g(\eta)=\frac{f^{\prime \prime}(\eta)}{f(\eta)}+\frac{2 c}{\eta}
$$

is an entire function. We show below that there exist squares $S_{N}$ with vertices $\pm R_{N} \pm \mathrm{i} R_{N}$ on which the maximum modulus of $g$ tends to zero as $R_{N} \rightarrow \infty$. Thus, in fact $g(\eta) \equiv 0$, and

$$
f^{\prime \prime}+\frac{2 c f}{\eta}=0
$$

This is of course simply (44) with $c=\sigma_{\text {ext }}$. Thus, the solution to the inner discrete problem is unique up to a scaling factor $c$, which is arbitrary, and which must be determined by matching.

In order to prove that $g$ vanishes, we produce squares $S_{N}$ with vertices $\pm R_{N} \pm \mathrm{i} R_{N}$ on which the maximum modulus of $g$ tends to zero as $R_{N} \rightarrow \infty$. We first need some bounds 
on the $\eta_{i}$, and we arrange them (as has been implicit throughout) such that $\eta_{1}<\eta_{2}<\cdots$. To obtain these bounds we need the following:

Lemma. If $p<n$ then

$$
\eta_{n}-\eta_{p}>\frac{(n-p)(n-p+2)}{4 c} \text { and } \eta_{n}>\frac{n^{2}}{4 c}
$$

Proof. Summing the original equation from $i=1$ to $m$ we have

$$
\sum_{i=1}^{m} \frac{1}{\eta_{i}}=\sum_{i \leqslant m} \sum_{j \geqslant m+1} \frac{1}{\eta_{j}-\eta_{i}} .
$$

If $p \leqslant m<n$ then

$$
c>\sum_{i=1}^{m} \frac{1}{\eta_{i}}>\sum_{p \leqslant i \leqslant m} \sum_{m+1 \leqslant j \leqslant n} \frac{1}{\eta_{j}-\eta_{i}} \geqslant \frac{(n-m)(m-p+1)}{\eta_{n}-\eta_{p}} .
$$

Choosing $m$ to maximise this (about $(n+p) / 2)$ we have

$$
c>\frac{(n-p)(n-p+2)}{4\left(\eta_{n}-\eta_{p}\right)}
$$

as required. Then $c>1 / \eta_{1}$ so $\eta_{1}>1 / c$ which gives

$$
\eta_{n}>\frac{n^{2}}{4 c}
$$

Now, given $N$ we know by the Lemma that

$$
\eta_{2 N}-\eta_{N} \geqslant \frac{N(N+2)}{4 c}
$$

so some $r$ in $N \leqslant r \leqslant 2 N-1$ has

$$
\eta_{r+1}-\eta_{r} \geqslant \frac{N}{4 c} \text {. }
$$

Let $R_{N}=\left(\eta_{r}+\eta_{r+1}\right) / 2$. Then $R_{N}>\eta_{r} \geqslant \eta_{N}>N^{2} / 4 c$. For $\eta$ on the top, left or bottom edges of $S_{N},\left|\eta-\eta_{n}\right| \geqslant\left(|\eta|+\eta_{n}\right) / 3$ so

$$
\left|\sum_{n=1}^{\infty} \frac{1}{\eta-\eta_{n}}\right| \leqslant 3 \sum_{n=1}^{\infty} \frac{1}{|\eta|+\eta_{n}} \leqslant 3 \sum_{n=1}^{\infty} \frac{1}{|\eta|+n^{2} / 4 c}=O\left(N^{-1}\right) .
$$

For $z$ on the right edge of $S_{N},\left|\eta-\eta_{n}\right| \geqslant\left|R_{N}-\eta_{n}\right|$. For $n \geqslant r+1$,

$$
\left|R_{N}-\eta_{n}\right|=\left(\eta_{n}-\eta_{r+1}\right)+\left(\eta_{r+1}-R_{N}\right) \geqslant \frac{(n+(r+1))^{2}}{4 c}+\frac{N}{8 c}
$$

so that

$$
\sum_{n=r+1}^{\infty} \frac{1}{\left|\eta-\eta_{n}\right|} \leqslant \sum_{i=0}^{\infty} \frac{1}{N / 8 c+i^{2} / 4 c}=O\left(N^{-1 / 2}\right)
$$


A similar argument shows that

$$
\sum_{1}^{r} \frac{1}{\left|\eta-\eta_{n}\right|}=O\left(N^{-1 / 2}\right)
$$

so that

$$
\frac{f^{\prime}}{f} \rightarrow 0 \text { on } S_{N} \quad \text { as } N \rightarrow \infty .
$$

The same argument can be applied to

$$
\sum_{i=1}^{\infty} \frac{1}{\left(\eta-\eta_{i}\right)^{2}}
$$

so that in fact $g \rightarrow 0$ on $S_{N}$ as $N \rightarrow \infty$ as required.

\section{Conclusions}

The systematic asymptotic study of the well-known problem of a pile-up against a locked dislocation poses interesting analytical and numerical challenges. Also, any approximate theory for large numbers of dislocations can be tested against the exact results of Eshelby et al. (1951).

We have been able to show that a continuum approximation is valid away from the head and the tail of the pile-up and that this approximation can be matched with inner regions in which there is no continuum density. In the more important region near the head of the pile-up, the lowest order inner problem comprises an infinite set of nonlinear algebraic equations for the dislocation positions and this system of equations must be truncated carefully if the correct solution is to be obtained numerically.

The sequel will describe precisely the same formulation for the more practically important case of a pile-up against an interface in a bimetallic solid, for which the continuum density is known, but the function corresponding to $q$ in (8) is not. This analysis will show that, although (3) and (50) and the corresponding results for a double-ended pileup suggest that the force on any locked dislocation supporting a pile-up of $n$ dislocations in a uniform material might be approximately proportional to $n$ as the number of dislocations increases, this is not the case for the force on an interface supporting a pile-up in a bimetallic solid.

\section{Acknowledgements}

Valuable discussions with Dr. S.G. Roberts, Dr. A.J. Wilkinson, and Prof. L.M. Brown are gratefully acknowledged. This work was supported by EPSRC under Grant GR/ S34045/01.

\section{References}

Brown, L.M., 2005. Dislocation pile-ups, slip-bands, ellipsoids, and cracks. Mater. Sci. Eng. A 400-401, 2-6. Cahn, R.W., Haasen, P. (Eds.), 1996. Physical Metallurgy, vol. 3, fourth ed. North-Holland, Amsterdam. 
Eshelby, J.D., Frank, F.C., Nabarro, F.R.N., 1951. The equilibrium of linear arrays of dislocations. Philos. Mag. $42,351-364$.

Hinch, E.J., 1991. Perturbation Methods. Cambridge University Press, Cambridge, MA.

Hirth, J.P., Lothe, J., 1992. Theory of Dislocations, 2nd ed. Krieger Publishing, Malabar, FL.

Leibfried, G., 1951. Verteilung von Versefzungen im statischen Gleichgewicht. Zeitschrift für Physik 130, 214-226.

Ockendon, H., Ockendon, J.R., 1983. Dynamic dislocation pile-ups. Philos. Mag. A 47, 707-719. 